\title{
Defectos eritrocíticos y densidad de la parasitemia en pacientes con malaria por Plasmodium falciparum en Buenaventura, Colombia
}

\author{
Martha Moyano y Fabián Méndez²
}

Forma de citar

Moyano M, Méndez F. Defectos eritrocíticos y densidad de la parasitemia en pacientes con malaria por Plasmodium falciparum en Buenaventura, Colombia. Rev Panam Salud Publica. 2005;18(1):25-32.

RESUMEN Objetivos. Determinar la prevalencia de algunos defectos eritrocíticos y evaluar su relación con la densidad de la parasitemia en personas con diagnóstico de malaria (paludismo) por Plasmodium falciparum en una población del Pacífico colombiano.

Métodos. Se realizó un estudio de prevalencia en 242 personas con malaria por P. falciparum que consultaron al Programa de Enfermedades Tropicales en la ciudad de Buenaventura, Colombia. Se midieron los niveles de parasitemia y se determinó la presencia de defectos eritrocíticos congénitos (deficiencia de glucosa-6-fosfato deshidrogenasa [G6PD] y presencia de hemoglobinas anormales y de talasemias) y de otros factores potencialmente relacionados con los niveles de parasitemia.

Resultados. La prevalencia obtenida de defectos eritrocíticos fue de $26,4 \%$ (IC95\% 21,0 a $32,5)$, similar a la hallada en estudios realizados antes en la misma zona. En los modelos de regresión múltiple, las personas con drepanocitosis o deficiencia total de la G6PD presentaron una menor densidad de parasitemia que las personas sin defecto, y el riesgo de parasitemias altas fue menor en estas personas después de ajustes respecto a otras variables de interés (razón de posibilidades [odds ratio, RP]: 0,30 y 0,72 , respectivamente).

Conclusiones. Los resultados confirman una alta prevalencia de defectos eritrocíticos en el Pacífico colombiano, en una población con características étnicas similares a las de algunas poblaciones del África Occidental, y aportan información en favor de la existencia de resistencia innata a la malaria en personas portadoras de hemoglobina AS o con deficiencia de la G6PD.

Palabras clave Malaria, Plasmodium falciparum, hemoglobinopatías, anemia de células falciformes, glucosafosfato deshidrogenasa.

Escuela de Odontología, Universidad del Valle, Cali, Colombia.

2 Escuela de Salud Pública, Universidad del Valle, Cali, Colombia. Toda correspondencia deberá dirigirse a: Fabián Méndez, Calle 4B \# 36-00, Edificio 118, Escuela de Salud Pública, Universidad del Valle, Sede San Fernando. Tel.: (57 2) 554 2476. Fax: (57 2) 557 0425. Correo electrónico: famendez@ univalle.edu.co
La malaria, o paludismo, es la enfermedad más importante entre las transmitidas por vectores en las regiones tropicales del mundo. En América se estima que viven aproximadamente 175 millones de personas en algún riesgo de contraer esta enfermedad, y de ellas $22 \%$ se registraron en Colombia durante los años 2001 y 2002. En ese último año se produjo un incremento de más de $8 \%$ en la incidencia con respecto a $2001(1,2)$.

Se ha señalado repetidamente una coincidencia geográfica entre la pre- 
sencia de malaria y de defectos eritrocíticos como las hemoglobinopatías (hemoglobinas anormales, talasemias) y la deficiencia de deshidrogenasa de glucosa-6-fosfato (G6PD), que es el trastorno metabólico asociado con mayor frecuencia con la anemia hemolítica (3). En la costa del Pacífico colombiano, región con malaria endémica, se ha señalado una alta prevalencia de tales defectos. En 1995 se ubicó en $15 \%$ la prevalencia de hemoglobinopatías en las poblaciones de Guapi, Timbiquí, Noanamito y Zaragoza (4), y en 1984 se calculó en 13,7\% la prevalencia de deficiencia de G6PD en el corregimiento del Bajo Calima, situado en la parte noroccidental del municipio de Buenaventura (5).

La coincidencia geográfica mencionada apoya la teoría de que la malaria es un factor selectivo respecto a la presencia de defectos eritrocíticos. En el caso específico de la deficiencia de G6PD, las mujeres heterocigotas portadoras de esta deficiencia podrían estar protegidas contra la malaria causada por Plasmodium falciparum y las hemoglobinopatías confieren algún grado de protección contra la malaria grave por $P$. falciparum $(4,6,7)$. Algunos estudios realizados en poblaciones africanas han mostrado una menor densidad de la parasitemia, que pareciera indicadora de esa protección o resistencia (8-10), mientras que otros parecen indicar que los defectos eritrocíticos innatos no confieren protección contra la malaria (11). Aunque el mecanismo de protección de las células portadoras de alguno de los defectos eritrocíticos ha sido bien documentado en estudios in vitro (12-14), tal protección no se ha encontrado en estudios de tipo poblacional (15). En general, la información obtenida indica que la presencia de defectos eritrocíticos pudiera redundar en una menor incidencia de malaria por P. falciparum, con la posibilidad de que las personas con estos defectos tengan una parasitemia menos densa.

El objetivo de esta investigación fue establecer la prevalencia de defectos eritrocíticos y evaluar su relación con la presencia de una parasitemia de menor densidad en personas con diag- nóstico de malaria en la zona urbana del municipio de Buenaventura, población colombiana que tiene características étnicas en común con las de África Occidental. El presente estudio aspira a contribuir a la comprensión del mecanismo evolutivo que propone a la malaria como un factor de presión selectiva sobre los defectos eritrocíticos, del mismo modo que se ha propuesto en el caso de esas poblaciones africanas.

\section{MATERIALES Y MÉTODOS}

\section{Población de estudio y métodos}

La investigación se efectuó entre los meses de julio y septiembre de 2001, en el municipio de Buenaventura, situado en la zona tropical húmeda de la costa del Pacífico en el departamento del Valle del Cauca, Colombia. El municipio tiene cerca de 300000 habitantes, de los cuales $85 \%$ viven en la zona urbana. En 1999 el municipio registró $86,3 \%$ de los casos de malaria del departamento del Valle, 58,8\% de lo cuales fueron causados por $P$. falciparum (16).

Se calculó un tamaño muestral de 240 , suponiendo para la zona del estudio una prevalencia de defectos eritrocíticos de $20 \%(5,17,18)$ y estimando, de los estudios realizados en África Occidental (19), una incidencia de 10 y 30\% de parasitemia "alta" (1 000 formas anulares o más por microlitro de sangre) en personas con defectos eritrocíticos y sin ellos, respectivamente. Los sujetos del estudio se seleccionaron entre las personas que consultaron a los servicios del laboratorio especializado del Programa de Enfermedades Tropicales (PET), principal centro de diagnóstico en el municipio. Ingresaron en el estudio los que cumplían los siguientes criterios: resultado positivo a $P$. falciparum en el análisis de gota gruesa, edad mayor de 1 año, residencia temporal o permanente en el municipio de Buenaventura y aceptación a participar mediante la firma de una carta de consentimiento. Se excluyó a las personas con infecciones parasitarias mixtas y diferentes de ma- laria por P. falciparum, y a aquellas con malaria grave, ya que en esta situación el secuestro de parásitos es frecuente y el nivel de parasitemia puede no reflejar el deterioro ni la respuesta inmunitaria del paciente.

\section{Exposición de interés}

Como principal exposición de interés se consideró la presencia de uno o más de los siguientes defectos eritrocíticos: deficiencia de G6PD, hemoglobinas anormales y talasemias. La prueba de la deficiencia de G6PD se realizó en las 8 horas posteriores a la obtención de la muestra, según el método visual práctico de Motulsky y CampbellKraut (20), preparado por Sigma ${ }^{\circledR}$ en el Kit Procedure $\mathrm{N}^{\circ}$ 400. De las muestras de sangre se obtuvieron además los valores hematológicos de hemoglobina y hematócrito. Las muestras se almacenaron a $4{ }^{\circ} \mathrm{C}$ hasta el diagnóstico de hemoglobinas anormales y talasemias mayores, mediante electroforesis de celulosa a un pH de 7,8, y la cuantificación de hemoglobinas A2 y fetal $(21,22)$ se efectuó en el laboratorio especializado de hematología de la Universidad del Valle, en Cali.

\section{Otras variables de exposición}

Mediante una encuesta estructurada, validada con anterioridad, se evaluaron otras exposiciones que pudieran relacionarse con la densidad de la parasitemia. Las variables se clasificaron en: sociodemográficas (edad, sexo, etnia, lugar de residencia, ocupación, escolaridad), tiempo de evolución de la enfermedad; síntomas de malaria, antecedentes de esta, automedicación, nivel de conocimientos sobre la enfermedad y uso de medidas preventivas. De acuerdo con los valores de hemoglobina calculados para zonas con una altitud de 50 metros sobre el nivel del mar (23), la anemia al momento de la consulta se definió como un valor de hemoglobina menor de $11,6 \mathrm{~g} / \mathrm{dL}$ en los niños menores de 13 años, menor de $12,5 \mathrm{~g} / \mathrm{dL}$ en las mujeres y menor de $14 \mathrm{~g} / \mathrm{dL}$ en los varones. 


\section{Variable de resultado}

La densidad de la parasitemia se calculó mediante la técnica de gota gruesa (23). El valor se obtuvo de la cuenta de parásitos con tinción de Giemsa en 200 leucocitos y la densidad de la parasitemia se calculó tomando como norma una concentración de 8000 leucocitos por microlitro $(\mu \mathrm{L})$ de sangre (24).

\section{Administración de datos y métodos estadísticos}

La información obtenida se introdujo en una base de datos construida con el programa Epi Info $6.04 \mathrm{~d}^{3}$ y el análisis estadístico se realizó con el programa Stata $6.0^{\mathrm{TM}}$. $^{4}$

Las variables continuas se sometieron a las transformaciones necesarias para poder utilizar los métodos basados en el modelo gaussiano de probabilidad, y en el caso de las variables categóricas se obtuvieron las frecuencias y proporciones. Se estimó la prevalencia de cada uno de los defectos eritrocíticos y se calcularon los intervalos de confianza de $95 \%$ binomiales correspondientes.

En el análisis categórico de la densidad de la parasitemia se utilizó como punto de corte la cifra de 1000 formas anulares por $\mu \mathrm{L}$ de sangre, porque se encontró una asociación positiva significativa entre valores superiores a 1000 formas por $\mu \mathrm{L}$ y la presencia de síntomas graves de malaria, como escalofrío y vómito. Se realizó análisis de asociación entre la exposición de interés (defecto eritrocítico de naturaleza innata) y la variable de efecto (densidad de la parasitemia). Se evaluaron posibles variables de confusión mediante la construcción de tablas de $2 \times 2$ estratificadas y la estimación de las razones de posibilidades (odds ratio,

\footnotetext{
3 Programa estadístico gratuito provisto por los Centers for Disease Control and Prevention (DCD), de Atlanta, Georgia (http://www.cdc. gov/epiinfo/).

4 Versión 6.0 (1999). Stata Corporation, 4905 Lakeway Drive, College Station, Texas 77845 (http:/ / www.stata.com/).
}

RP) comunes de Mantel y Haenszel, con sus respectivos intervalos de confianza del 95\%. Para valorar la significación estadística se utilizó la prueba exacta de Fisher $(P<0,05$, bilateral). Además, para evaluar confusión e interacción se realizó un análisis de regresión múltiple utilizando un modelo logístico en el que la variable de respuesta fue la densidad de la parasitemia, con un punto de corte de 1000 formas anulares por $\mu \mathrm{L}$. Para obtener un modelo de regresión múltiple reducido, se realizó al principio una selección de variables en cada una de las categorías de exposición siguientes: defectos eritrocíticos, características sociodemográficas, presencia y duración de síntomas, concentraciones de hemoglobina y hematócrito, antecedentes de malaria, automedicación, y conocimientos y prácticas, teniendo en cuenta la significación estadística y biológica para prevenir la enfermedad. A continuación se seleccionaron las variables apropiadas entre todas las que se encontraron asociadas en cada categoría. Para elegir las variables en la construcción del modelo final se utilizó un nivel de significación menor de 0,20 (25). El análisis de regresión se realizó entre todas las personas incluidas en el estudio y mediante un subanálisis en las personas con una densidad de parasitemia menor de 10000 formas anulares por $\mu \mathrm{L}$ de sangre, ya que la densidad media de la parasitemia en personas con malaria en el $\mathrm{Pa}$ - cífico colombiano se encuentra alrededor de ese valor (26); además, las personas con una parasitemia de esa densidad constituyeron 76,9\% (186/ 242) de la población del estudio.

Además, por medio de regresión logística politómica se efectuó un análisis de asociación en el que las categorías de la densidad de la parasitemia se ampliaron a: <1 000, $1000-$ 5 000, 5 001-10 000 y >10 000 parásitos por $\mu \mathrm{L}$ de sangre.

\section{RESULTADOS}

El estudio se realizó entre los meses de julio y septiembre de 2001, período en que se incorporaron 242 personas, en su mayoría varones $(153 / 242=$ $63,2 \%)$, con un promedio de edad de 27,8 años.

La prevalencia de defectos eritrocíticos innatos fue de $26,4 \%$. En particular, se observó una prevalencia de $12 \%$ de la deficiencia de G6PD, y de 14,9\% de hemoglobinas anormales. La hemoglobinopatía AS, o rasgo falciforme, fue la de mayor prevalencia $(7 \%)$, seguida de las hemoglobinas AC $(6,2 \%)$ y $\mathrm{AD}(1,7 \%)$ (cuadro 1$)$. El promedio de la densidad de la parasitemia fue de 7570 formas anulares por $\mu \mathrm{L}$ de sangre (desviación estándar: 13 428), y $40,5 \%$ de las personas estudiadas tuvieron una densidad de parasitemia menor de 1000 formas anulares por $\mu \mathrm{L}$ de sangre.

CUADRO 1. Prevalencia de defectos eritrocíticos innatos en personas con malaria por Plasmodium falciparum que consultaron al Programa de Enfermedades Tropicales. Julio a septiembre de 2001, Buenaventura-Valle, Colombia

\begin{tabular}{lccc}
\hline \multicolumn{1}{c}{ Tipo de defecto } & Frecuencia & $\begin{array}{c}\text { Prevalencia } \\
\%\end{array}$ & IC95\% ${ }^{\mathrm{a}}$ \\
\hline Deficiencia de G6PD & 29 & 12,0 & $8,2-16,8$ \\
Deficiencia intermedia de G6PD & 13 & 5,4 & $2,9-9,0$ \\
Deficiencia total de G6PD & 16 & 6,6 & $3,8-10,5$ \\
Hemoglobinas anormales & 36 & 14,9 & $10,6-20,0$ \\
Hemoglobina AS-drepanocitosis & 17 & 7,0 & $4,2-11,0$ \\
Otras hemoglobinopatías (AD, AC) & 19 & 7,9 & $4,8-12,0$ \\
Total de defectos eritrocíticos & $64^{\mathrm{c}}$ & 26,4 & $21,0-32,5$ \\
\hline
\end{tabular}

a IC95\%: intervalo de confianza de 95\%.

b G6PD: deshidrogenasa de glucosa-6-fosfato.

${ }^{c}$ Una persona tuvo simultáneamente deficiencia intermedia de G6PD y hemoglobina anormal. 
CUADRO 2. Asociación entre defectos eritrocíticos innatos y una densidad de parasitemia de $\geq 1000$ formas anulares por microlitro de sangre. Buenaventura, 2001

\begin{tabular}{lccccc}
\hline & \multicolumn{2}{c}{ Parasitemia } & & \\
\cline { 2 - 3 } \multicolumn{1}{c}{ Defecto } & $\begin{array}{c}\geq 1000 / \mu \mathrm{L} \\
\text { No. }(\%)\end{array}$ & $\begin{array}{c}<1000 / \mu \mathrm{L} \\
\text { No. }(\%)\end{array}$ & RPa & IC95\% ${ }^{\mathrm{b}}$ \\
\hline Deficiencia de G6PD & & & & \\
$\quad$ Ninguna deficiencia & $110(87,3)$ & $68(85,0)$ & 1 & - \\
Deficiencia de cualquier tipo & $16(12,7)$ & $12(15,0)$ & 0,82 & $0,37-1,82$ \\
Deficiencia de tipo intermedio & $7(5,6)$ & $5(6,2)$ & 0,87 & $0,28-2,69$ \\
Deficiencia de tipo completo & $9(7,1)$ & $7(8,8)$ & 0,79 & $0,29-2,16$ \\
Hemoglobinas anormales & & & & \\
Ninguna hemoglobina anormal & $110(86,6)$ & $68(79,1)$ & 1 & - \\
Alguna hemoglobina anormal & $17(13,4)$ & $18(20,9)$ & 0,58 & $0,28-1,20^{\mathrm{d}}$ \\
Hemoglobina AS & $6(4,7)$ & $11(12,8)$ & 0,34 & $0,12-0,92^{\mathrm{e}}$ \\
Hemoglobina AC & $9(7,1)$ & $5(5,8)$ & 1,11 & $0,37-3,30$ \\
Hemoglobina AD & $2(1,6)$ & $2(2,3)$ & 0,62 & $0,11-3,59$ \\
Defectos en general & & & & \\
Ninguno & $110(76,4)$ & $68(69,4)$ & 1 & - \\
Alguno & $34(23,6)$ & $30(30,6)$ & 0,70 & $0,39-1,24^{\mathrm{e}}$ \\
\hline
\end{tabular}

a $\mathrm{RP}$, razón de posibilidades (odds ratio).

b IC95\%: intervalo de confianza de $95 \%$.

c G6PD: deshidrogenasa de glucosa-6-fosfato.

d $0,05<P<0,25$.

e $P<0,05$.

CUADRO 3. Análisis multifactorial del riesgo de parasitemia de $\geq 1000$ formas anulares por microlitro de sangre, según el tipo de defecto y otras exposiciones de interés. Buenaventura, Colombia, 2001

\begin{tabular}{|c|c|c|c|}
\hline Variables & $\mathrm{RP}^{\mathrm{a}}$ ajustada & IC95\% ${ }^{b}$ & Valor $P$ \\
\hline \multicolumn{4}{|l|}{ Defectos eritrocíticos } \\
\hline Ningún defecto & 1 & & \\
\hline Deficiencia intermedia de G6PDC & 1,51 & $0,37-6,14$ & 0,561 \\
\hline Deficiencia total de G6PD & 0,72 & $0,23-2,19$ & 0,557 \\
\hline Drepanocitosis (hemoglobina AS) & 0,30 & $0,09-0,99$ & 0,048 \\
\hline Hemoglobina AC & 1,11 & $0,33-3,70$ & 0,864 \\
\hline Hemoglobina AD & 0,51 & $0,06-4,34$ & 0,538 \\
\hline \multicolumn{4}{|l|}{ Sociodemográficas } \\
\hline Sexo femenino & 1,09 & $0,58-2,03$ & 0,786 \\
\hline Raza negra & 1,50 & $0,70-3,22$ & 0,294 \\
\hline Residencia rural & 2,19 & $1,12-4,29$ & $0,023^{d}$ \\
\hline Tiempo de residencia $\geq 1$ año & 0,87 & $0,39-1,98$ & 0,748 \\
\hline \multicolumn{4}{|l|}{ Edad (años): } \\
\hline $1-10$ & 1 & & \\
\hline $11-20$ & 0,47 & $0,16-1,39$ & 0,173 \\
\hline 21-40 y ocupación urbana & 0,77 & $0,24-2,53$ & 0,672 \\
\hline 21-40 y ocupación rural & 0,55 & $0,17-1,78$ & 0,316 \\
\hline$>40$ & 0,51 & $0,16-1,59$ & 0,245 \\
\hline \multicolumn{4}{|l|}{$\begin{array}{l}\text { Síntomas clínicos y hematológicos, } \\
\text { episodios de malaria }\end{array}$} \\
\hline Escalofríos & 4,60 & $1,06-19,89$ & $0,041^{d}$ \\
\hline Anemia & 0,60 & $0,26-1,35$ & 0,218 \\
\hline Días de enfermedad $>7$ & 0,36 & $0,19-0,71$ & $0,003^{d}$ \\
\hline Otro episodio de malaria & 0,94 & $0,49-1,80$ & 0,850 \\
\hline \multicolumn{4}{|l|}{ Automedicación y conocimientos } \\
\hline \multicolumn{4}{|l|}{ Uso de antimalárico o antibiótico } \\
\hline en el último mes & 0,86 & $0,40-1,86$ & 0,699 \\
\hline Conocimiento de los síntomas & 0,72 & $0,40-1,29$ & 0,267 \\
\hline Conocimiento de los antimaláricos & 0,51 & $0,22-1,19$ & $0,120^{e}$ \\
\hline
\end{tabular}

En el cuadro 2 se resumen los datos del análisis de asociación unifactorial entre los defectos eritocíticos y la parasitemia. Resalta una asociación negativa significativa entre la presencia de hemoglobina AS y la de parasitemias mayores de 1000 formas anulares por $\mu \mathrm{L}$ de sangre. Las personas con este tipo de defecto mostraron una probabilidad $66 \%$ menor de presentar parasitemias altas que aquellas sin ningún defecto eritrocítico $(\mathrm{RP}=0,34)$. Nuestros datos apuntan a una tendencia hacia una menor parasitemia en presencia de la mayor parte de los otros defectos estudiados, si bien en ninguno de ellos el valor $P$ fue menor de 0,05 .

En el análisis multifactorial (cuadro 3), después del ajuste, se conservó la fuerte asociación negativa $(<1)$ entre la drepanocitosis y una densidad de parasitemia mayor de 1000 formas anulares por $\mu \mathrm{L}$. Además, las personas con un tiempo de evolución de la enfermedad mayor de siete días y aquellas con conocimiento de alguno de los antimaláricos de uso frecuente (cloroquina, sulfadoxina-pirimetamina y primaquina) mostraron una menor probabilidad de tener una densidad de parasitemia de $1000 / \mu \mathrm{L}$ o más, aunque esta última asociación no fue estadísticamente significativa. Los sujetos con anemia mostraron también una menor probabilidad de tener parasitemias altas. En cambio, las personas residentes en la zona rural y las que tuvieron escalofríos durante la enfermedad mostraron una mayor probabilidad de tener una densidad de parasitemia de 1000 formas anulares por $\mu \mathrm{L}$ o más. El modelo de regresión logística final (cuadro 4) indicó que la drepanocitosis era el único defecto congénito que permanecía vinculado con la densidad de la parasitemia. De igual manera, se observó que un tiempo de evolución de la enfermedad mayor de 7 días y el tener conocimiento de los medicamentos antimaláricos mantenían una relación negativa con parasitemias mayores de 1000 formas anulares por $\mu \mathrm{L}$, mientras que la residencia en zona rural y la presencia de escalofríos mostraron una asociación positiva.

De otra parte, el análisis politómico realizado por más de dos categorías de 
CUADRO 4. Modelo multifactorial final de factores asociados con una parasitemia $\geq 1000$ formas anulares en casos de malaria por $P$. falciparum. Buenaventura, Colombia, 2001

\begin{tabular}{lccc}
\hline \multicolumn{1}{c}{ Variable } & RPa ajustada & IC95\% & Valor $P$ \\
\hline Drepanocitosis (Hb AS) & 0,39 & $0,13-1,15$ & $0,089^{\mathrm{c}}$ \\
Días de enfermedad $>7$ & 0,37 & $0,20-0,68$ & $0,001^{\mathrm{d}}$ \\
Escalofríos & 3,92 & $0,98-15,77$ & $0,054^{\mathrm{c}}$ \\
Residencia rural & 1,96 & $1,09-3,52$ & $0,025^{\mathrm{d}}$ \\
Conoce antimaláricos & 0,53 & $0,25-1,15$ & $0,107^{\mathrm{c}}$ \\
\hline
\end{tabular}

a RP: razón de disparidad (odds ratio).

b IC95\%: intervalo de confianza de 95\%.

${ }^{c} P<0,05$.

d $0,05<P<0,20$

densidad de parasitemia como variable de interés apuntó a una asociación negativa entre la presencia de los defectos eritrocíticos y la de densidades altas en los intervalos de parasitemia menores de 10000 formas anulares por $\mu \mathrm{L}$. En esos intervalos de parasitemia, las RP estimadas por regresión logística politómica de las parasitemias mayores estuvieron por debajo de la unidad en los defectos evaluados. Específicamente, si se toma como categoría de referencia el grupo de personas con parasitemias menores de $1000 /$ $\mu \mathrm{L}$, las RP de parasitemias de 1000 a 5000 y de 5001 a 10000 formas anulares por $\mu \mathrm{L}$ resultan ser de 0,74 ( $P=$ $0,182)$ y $0,51(P=0,130)$, respectivamente. El riesgo estimado de las parasitemias mayores de 10000 indica una pérdida de la asociación señalada entre los defectos y la parasitemia alta $(\mathrm{RP}=$ $3,17 ; P>0,2)$, a excepción de la drepanocitosis, en la que se conservó un efecto protector $(\mathrm{RP}=0,54, P=0,148)$.

\section{DISCUSIÓN}

En este estudio, realizado en la costa del Pacífico colombiano, se encontró una prevalencia de defectos eritrocíticos innatos de $26,4 \%$, lo que concuerda con los datos publicados de deficiencia de G6PD (5) y presencia de hemoglobina $S(17,18)$ en poblaciones de la misma zona.

La prevalencia de deficiencia de G6PD fue de $12 \%$, valor similar al de $13,5 \%$ notificado en la región del río San Juan del Chocó en 1968 (27), y al de $13,7 \% \pm 5,2 \%$ del Bajo Calima, encontrado en 1984 (5). De igual forma, la prevalencia de hemoglobina AS de 7\% fue similar a la notificada en 1994 en Salahonda, que fue de $10 \%$ (18). En el estudio se encontró también que 14,9\% de las personas tenían algún tipo de hemoglobina anormal, resultado similar al de $15 \%$ obtenido por Gover en 1996 (28) en un grupo de estudiantes de la costa del Pacífico colombiano, y al de $14,3 \%$ notificado por Bernal et al. en 1995 (29) en las islas de San Andrés y Providencia. En un estudio realizado en 1998 en niños de la ciudad de Cartagena se encontró una prevalencia de $10 \%$ de hemoglobinas anormales, con mayor frecuencia la AS (30). En el presente estudio no se encontraron personas con talasemia mayor.

Para el análisis de asociación con la densidad de la parasitemia se utilizó un punto de corte de 1000 formas anulares por $\mu \mathrm{L}$ de sangre. La decisión se fundamentó en la asociación estadística constatada entre parasitemias mayores de $1000 / \mu \mathrm{L}$ y la presencia de escalofríos y vómito, utilizados como indicadores de enfermedad malárica. Por añadidura, en poco más de $40 \%$ de la muestra (98/242) las parasitemias estuvieron por debajo de $1000 / \mu \mathrm{L}$, lo que facilitó las comparaciones entre los dos grupos y proporcionó la potencia estadística apropiada. Ante las posibles limitaciones de este análisis dicotómico, se efectuó un análisis politómico que permitió evaluar de manera alterna la hipótesis de interés; los resultados dieron respaldo adicional a la asociación observada entre los defectos y la densidad de la parasitemia. Uno de los principales datos de nuestro estudio es la asociación negativa de la drepanocitosis con las parasitemias altas (RP =0,30; IC95\%: 0,09 a 0,99). Este dato fue constante en todos los modelos de regresión logística múltiple evaluados, lo que apunta a un menor riesgo de aparición de enfermedad grave por $P$. falciparum en personas con drepanocitosis que en aquellas sin este tipo de defecto. Lo anterior concuerda con los resultados de estudios in vitro que han demostrado los mecanismos de protección bioquímicos que previenen el desarrollo del parásito (12).

De igual manera, el análisis de regresión logística politómica de las diferentes categorías de densidad de parasitemia mostró una asociación negativa entre la presencia de cada uno de los defectos eritrocíticos y la presencia de densidades altas, en los intervalos de parasitemias menores de $10000 / \mu \mathrm{L}$. En las parasitemias mayores de $10000 / \mu \mathrm{L}$ esta asociación negativa se conservó solo en la drepanocitosis y, en menor grado, en la deficiencia total de G6PD. El dato anterior posiblemente sea consecuencia de la inmunidad parcial que generan estos defectos y puede ser reflejo de que quienes los poseen tienden a tener menos manifestaciones clínicas con parasitemias similares. Solo cuando la concentración de parásitos supera umbrales específicos —en este caso mayores de 10000 parásitos por $\mu \mathrm{L}$ de sangre - las personas con la mayor parte de estos defectos congénitos tienden a tener síntomas y a consultar más a los servicios de salud. El efecto protector de los defectos eritrocíticos innatos se sospechaba desde el decenio de 1960 (31), cuando empezó a observarse una mayor prevalencia de ellos en regiones con una alta prevalencia de malaria. Tal asociación recibió el apoyo de teorías como la hipótesis de Healdane (12), quien sostenía un mecanismo de coexistencia evolutiva entre algunos defectos genéticos heterocigotos presentes en el varón humano en regiones donde la malaria está presente como mecanismo de supervivencia. Se han efectuado muchas investigaciones encaminadas a comprobar esa teoría. Algunos resultados indican que las personas con esos defectos que viven en zonas maláricas poseen algún tipo de resistencia a la enfermedad, pero esos 
datos se han refutado en otros estudios en que se evalúa la protección contra la malaria común o sus formas graves. Estudios como el de Ruwende et al., realizado en 1995 en niños, muestran una relación entre la forma de deficiencia más común (G6PDA-) y una reducción de 46 a $58 \%$ del riesgo de malaria grave en mujeres heterocigotas y varones hemicigotos, si bien no ofrecen resultados concluyentes respecto a diferencias en las densidades de parasitemia (32).

$\mathrm{Al}$ evaluar la relación de otras variables de exposición con la densidad de la parasitemia se observa una asociación negativa entre el momento de búsqueda de atención y la presencia de densidades de parasitemia mayores de 1000 formas anulares por $\mu \mathrm{L}$ de sangre $(\mathrm{RP}=0,43)$. Este dato podría explicarse en parte por la ocurrencia de automedicación en los pacientes que consultan tarde o por la posible infección por parásitos menos virulentos que originan cuadros clínicos menos sintomáticos y de evolución más prolongada. Nuestros resultados indican además que el antecedente de un acceso de malaria no modifica, en las condiciones de la zona del estudio, la probabilidad de tener nuevos accesos con parasitemias altas. Lo anterior podría ser un indicio de la inestabilidad de la malaria en esta región, donde la baja premunición (inmunidad débil coexistente con el parásito) alcanzada por la población no modifica el riesgo esencial de sufrir malaria con parasitemias altas. En el presente estudio se observó también que las personas con anemia tenían una menor probabilidad de presentar una densidad de parasitemia de 1000 o más formas anulares por $\mu \mathrm{L}$, lo que concuerda con la asociación negativa, señalada en otros estudios, entre la presencia de hierro y la respuesta inmunitaria a la enfermedad. Específicamente, algunos estudios indican que el tratamiento oral con hierro en poblaciones con deficiencia se ha relacionado con una exacerbación aguda de determinadas infecciones, en particular la malaria (33-35). Además, en un estudio realizado en 1993 en la zona urbana de Buenaventura, Carrasquilla encontró un riesgo mayor de malaria en las personas con una alimentación rica en hierro (36). En el estudio presente, en concordancia con otro realizado en el suroeste de Nigeria (37), se ha observado que los residentes de zonas rurales tienden más a presentar una parasitemia alta que los de zonas urbanas. Lo anterior podría relacionarse con muy diversos factores que caracterizan la transmisión de la malaria en las zonas rural y urbana, como diferencias en la virulencia de las cepas parasitarias circulantes, en los tipos de vectores y en variables relacionadas con la automedicación y la búsqueda de tratamiento (38-40). Mientras otros estudios han apuntado a una acción protectora de los defectos contra la malaria (32), el presente, realizado entre individuos con la enfermedad activa, parece indicar un efecto protector contra densidades altas de parasitemia.

Una limitación del presente estudio es que algunos análisis estadísticos no alcanzan la potencia suficiente porque el tamaño de la muestra no se calculó para estimar la asociación entre la densidad de la parasitemia y cada uno de los defectos eritrocíticos. Otro posible factor limitante de nuestras conclusiones es la mayor proporción de varones presente en la muestra del estudio, lo que refleja la distribución por sexo de la enfermedad en la región (40), pero podría afectar a la validez de nuestras conclusiones respecto al sexo femenino. Por otra parte, no se realizó un diagnóstico de talasemias menores y no fue posible estudiar estos defectos, si bien la prevalencia notificada de talasemias en poblaciones colombianas de raza negra es más baja que la de la mayor parte de las hemoglobinopatías estudiadas (3,7\%) (29).

Los resultados de este estudio confirman la presencia de defectos eritrocíticos en esta región malárica y señalan la necesidad de evaluar los efectos de algunos fármacos antimaláricos en personas con deficiencia de G6PD. Específicamente, se ha encontrado que en personas con las variantes de G6PD de mayor prevalencia, la G6PD A(presente en África) y la G6PD mediterránea, se produce una hemólisis grave después de la exposición a ciertos agentes, como las sulfas, la primaquina o incluso la quinina. En la va- riante G6PD A-, después de dos a cuatro días de primaquina oral, todos los signos, síntomas y características diagnósticas son consecuencia de una crisis hemolítica (41). En comunidades refugiadas en Paquistán en las que se ha constatado la presencia de G6PD, se evita la primaquina como agente terapéutico único contra la malaria, y se recomienda usarla solamente contra los gametocitos, a pesar de sus efectos adversos (42). Por otro lado, en países como México se recomienda no utilizar la primaquina en regiones con problemas de acceso a servicios médicos o en que estos no tienen la capacidad para identificar deficiencias de G6PD o efectos perjudiciales del fármaco (43).

El presente estudio llama la atención sobre la importancia del diagnóstico de este tipo de defectos innatos en niños de regiones maláricas. En Inglaterra, donde la inmigración de poblaciones de raza negra ha generado una alta prevalencia de talasemias, se ha diseñado un programa de salud pública encaminado a evitar la mortalidad infantil por crisis hemolíticas graves y la consiguiente hiperbilirrubinemia (44). A semejanza de lo observado en un estudio realizado con reclusos en Singapur (45), en el presente se advirtió que la mayoría de las personas no conocían su estado de enfermedad congénita, lo que podría hacerlas susceptibles a complicaciones debido a la falta de un diagnóstico oportuno.

En resumen, en concordancia con estudios anteriores realizados en la zona, esta investigación confirmó una prevalencia alta de varios defectos eritrocíticos y la asociación de algunos de ellos con una menor parasitemia entre personas con accesos sintomáticos de malaria.

Agradecimientos. Los autores agradecen a la Secretaría de Salud Departamental del Valle del Cauca y a la Fundación Carvajal el haber financiado la presente investigación; a los laboratorios de Hematología y Parasitología de la Universidad del Valle su cooperación en el procesamiento de las muestras, y muy en particular a todas las personas que accedieron a participar en este estudio. 


\section{REFERENCIAS}

1. Pan American Health Organization. Status report on malaria programs in the Americas (based on 2001 data). Washington, D.C.; 2002. (PAHO/ HCP/HCT/M217/02).

2. Pan American Health Organization. Status report on malaria programs in the Americas (based on 2002 data). Washington, D.C.; 2003.

3. Cavalli-Sforza Ll, Bondmer WF. The Genetics of Human Populations. New York: Dover Publications; 1999.

4. Colombia, Ministerio de Salud, Instituto Nacional de Salud. Hemoglobinopatías en Colombia. Informe Quincenal Epidemiológivo Nacional 1997; 2(12):171.

5. Ramírez CD. Determinación de la frecuencia de la deficiencia de glucosa-6-fosfato deshidrogenasa en la población del Bajo Calima. [Tesis]. Cali: Universidad del Valle; 1984.

6. Hill A. Malaria resístance genes: a natural selection. Trans Roy Soc Trop Med Hyg. 1992; 86:225-6, 232.

7. Cappadoro M, Garibaldi G, Obrien E, Turrini F, Mannu F, Ulliers D, et al. Early phagocytosis of glucose-6-phosphate dehydrogenase (G6PD) deficient erythrocytes parasitized by Plasmodium falciparum may explain malaria protection in G6PD deficiency. Blood. 1998; 92(7):2527-34

8. Luzzatto L, Usanga EA, Reddy Sh. Glucose-6phosphate dehydrogenase deficient red cell: resistance to infection by malarial parasites. Science. 1969;164:839-941

9. Bienzle U, Guggenmoos-Holzmann I, Luzzato L. Malaria and erythrocyte glucose-6phosphate dehydrogenase variants in West Africa. Am J Trop Med Hyg. 1979;28(4): 619-21.

10. Ademowo OG, Falusi AG, Mewoyeka OO. Prevalence of asymptomatic parasitaemia in an urban and rural community in South Western Nigeria. Cent Afr J Med. 1995;41(1):18-21.

11. Martin SK. The malaria/G6PD hypothesis revisited. Parasitol Today. 1994;10(7):251-2.

12. Friedman MJ, Trager W. Bioquímica de la resistencia a la malaria. Investig Cienc. 1981;56: 98-107.

13. Roth EF, Raventos-Suárez $\mathrm{C}$, Rinalde A, Nagel RL. Glucose-6-phosphate dehydrogenase deficiency inhibits in vitro growth of Plasmodium falciparum. Proc Natl Acad Sci USA. 1983;80:298-9.

14. Destro Bisol G, D’Aloja E, Spedini G, Acatena R, Giardina B, Pascali V. Resistance to falciparum malaria in alpha-thalassemia, oxidative stress, and hemoglobina oxidation. Am J Phys Anthropol. 1999;109(2):269-73.

15. Martin SK, Miller LH, Alling D, Okoye VC, Esan GJF, Osunkoya BO, et al. Severe malaria and glucose-6-phosphate dehydrogenase deficiency: a reappraisal of the malaria/G-6-PD hypothesis. Lancet. 1979;I:524-6.

16. Mendez F, Carrasquilla G, Muñoz A. Risk factors associated with malaria infection in an urban setting. Trans R Soc Trop Med Hyg. 2000; 94:367-71.

17. Cuervo AC. Determinación de la frecuencia del rasgo falciforme en la población de bajo Calima. [Tesis]. Cali: Universidad del Valle; 1984.
18. Pereira FD, Sáenz I. Hemoglobinopatías en niños. Colomb Med. 1996;27(34):146-9.

19. Bienzle U, Holzmann-Guggenmoos I, Luzzatto L. Malaria and erythrocyte glucose-6phosphate dehydrogenase variants in West Africa. Am J Trop Med Hyg. 1979;28(4): 619-21.

20. Organización Mundial de la Salud. Normalización de las técnicas de estudio de la G6PD. Ginebra: OMS; 1967. (Serie de Informes Técnicos No. 366.)

21. Sáenz GF, Moreira J, González R. Laboratorio hemoglobinopatías: manual latinoamericano. Costa Rica: CIHATA; 1980;1-155.

22. Evatt BL, Lewis SM, Lothe F, McArthur JR. Anemia: fundamental diagnostic hematology. Geneva: US Department of Health and Human Services and World Health Organization; 1983;1-121.

23. Cuéllar F, Restrepo A, Falabella F. Fundamentos de medicina: hematología, $5^{\mathrm{a}}$ ed. Medellín: Corporación para investigaciones biológicas; 1998.

24. World Health Organization. Assesment of therapeutic efficacy of antimalarial drugs for uncomplicated falciparum malaria in areas with intense transmission. Geneva: WHO; 1996. (WHO Report MAL/96.1077.)

25. Rothman KJ, Greenland S. Modern epidemiology, 2nd ed. Philadelphia: LippincottRaven Publishers; 1998.

26. Mendez F. Determinants of treatment response to sulfadoxine-pyrimethamine and subsequent transmision potential in falciparum malaria. [Tesis doctoral]. Baltimore: Johns Hopkins University; 2000.

27. Restrepo AM, Gutiérrez E. The frequency of glucose-6-phosphate dehydrogenase deficiency in Colombia. Am J Hum Genet. 1968 20:82-5.

28. Gover L. Prevalencia de hemoglobinopatías en la costa pacífica. Boletín Informativo de la Sociedad Colombiana de Genética. 1996; 2(1):3.

29. Bernal MP, Giraldo A, Bermúdez AJ, Moreno E. Estudio de la frecuencia de hemoglobinopatías en las islas de San Andrés y Providencia, Colombia. Biomedica. 1995;15:5-9.

30. Silva JR, Malambo D, Silva DF, Fals E, Fals O, Rey J. Tamizaje de hemoglobinopatías en una muestra de la población infantil de Cartagena. Pediatria. 1998; 33(2):86-9.

31. Allison AG. Glucose-6-phosphate dehydrogenase deficiency in red blood cells of East Africans. Nature. 1960;186:531-2.

32. Ruwende C, Khoo SC, Snow RW, Yates SNR, Kwiatkowski D, Gupta S, et al. Natural selection of hemi- and heterozygotes for G6PD deficiency in Africa by resistance to severe malaria. Nature. 1995; 376:246-9.

33. Murray MJ, Murray AB, Murray MB, Murray CJ. The adverse effect of iron repletion on the course of certain infections. Br Med J. 1978; 2(6145):1113-5.

34. Smith AW, Hendrickse RG, Harrison C, Hayes RJ, Greenwood BM. The effects on malaria of treatment of iron deficiency anemia with oral iron in Gambian children. Ann Trop Paediatr. 1989; 9(1):17-23.
35. Oppenheimer SJ. Iron and its relation immunity and infectious disease. J Nutr. 2001;131 (2S-2):633S-5S

36. Carrasquilla JG. Epidemiology of malaria in low endemic areas. [Tesis doctoral]. Cambridge (Massachussetts): Harvard University; 1993.

37. Ademowo OG, Falusi AG, Mewoyeka OO. Prevalence of asymtomatic parasitaemia in an urban and rural community in south western Nigeria. Cent Afr J Med. 1995;41(1):18-21.

38. Olano V, Carrasquilla G, Méndez F. Transmisión de la malaria urbana en Buenaventura, Colombia: aspectos entomológicos. Rev Panam Salud Publica. 1997;1(4):287-94.

39. Nieto T, Mendez F, Carrasquilla G. Knowledge, beliefs and practices relevant for malaria control in an endemic urban area of the Colombian Pacific. Soc Sci Med. 1999;49 (5):601-9.

40. Méndez F, Carrasquilla G. Epidemiología de la malaria en la zona urbana de Buenaventura: análisis de la ocurrencia en el período 1987-1993. Colomb Med. 1995;26(3):77-85.

41. Lukens JN. Glucose-6-phosphate dehydrogenase deficiency and related deficiencies involving the pentose phosphate pathway and glutathione metabolism. En: Lee GR, Bithell TC, Foerster J, Athens JW, Lukens JN, eds. Wintrobe's Clinical Hematology. Philadelphia: Lea \& Ferbirger; 1993:1006-22.

42. Bouma MJ, Goris GM, Akhtar T, Khan N, Khan N, Kita E, et al. Prevalence and clinical presentation of glucose-6-phosphate dehydrogenase deficiency in Pakistani Pathan and Afghani refugee communities in Pakistan; implications for the use of primaquine in regional malaria control programmes. Trans $\mathrm{R}$ Soc Trop Med Hyg. 1995;89:62-4.

43. Lopez FJ. Is primaquine useful and safe as true exo-erythrocytic merontocidal, hypnozoitocidal and gametocidal antimalarial drug? Salud Publica Mex. 1999;41:410-9.

44. Modell B, Khan M, Darlison M, King A, Layton M, Petrou M, et al. A national register for surveillance of inherited disorders: b-thalassaemia in the United Kingdom. Bull World Health Organ. 2001;79(8):704-12.

45. Lim MK, Tan EH, Wan A, Chao AK. Prevalence of G6PD deficiency among recruits in the Singapore Armed Forces. Ann Acad Med Singapore. 1995;2412:322-4.

Manuscrito recibido el 5 de marzo de 2004. Aceptado para publicación, tras revisión, el 25 de enero de 2005 
ABSTRACT Objectives. To determine the prevalence of some erythrocyte defects and to evaluate the relation that that has with parasitemia density in individuals diagnosed with Plasmodium falciparum malaria in a population in the Pacific coastal region of Colombia.

\section{Erythrocyte defects and parasitemia density in patients with Plasmodium falciparum malaria in Buenaventura, Colombia}

Keywords
Methods. This prevalence study was carried out with 242 persons with $P$. falciparum malaria who had gone for consultation at the Program of Tropical Diseases diagnostic center in the city of Buenaventura, Colombia. The parasitemia levels were measured, and also determined was the presence of congenital erythrocyte defects (glucose-6-phosphate dehydrogenase (G6PD) deficiency, abnormal hemoglobins, and thalassemias) and of other factors possibly related to parasitemia levels.

Results. The prevalence of erythrocyte defects was $26.4 \%$ (95\% confidence interval, $21.0 \%-32.5 \%$ ), which was similar to what had been found in previous studies in the same area of Colombia. In the multiple regression models, individuals with sickle cell anemia or a complete deficiency of G6PD had a lower density of parasitemia than did persons without any erythrocyte defect. After adjusting for other variables of interest, the risk of high parasitemias was lower in persons with sickle cell anemia (odds ratio $=0.30$ ) and individuals with a complete deficiency of G6PD (odds ratio $=0.72$ ).

Conclusions. Our results confirm the high prevalence of erythrocyte defects in Colombia's Pacific coastal region, in a population with ethnic characteristics that are similar to those of some populations in West Africa. Our results also lend support for the existence of innate resistance to malaria among carriers of hemoglobin AS and in persons with G6PD deficiency.

Malaria, Plasmodium falciparum, hemoglobinopathies, anemia, sickle cell trait, glucosephosphate dehydrogenase.

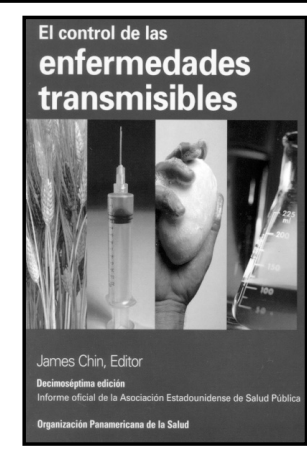

\section{El control de las enfermedades transmisibles, $17^{a}$ ed. ¡Ahora disponible en formato electrónico!}

Este libro es una obra de consulta ampliamente conocida sobre las enfermedades transmisibles. Fácil de entender y de usar, contiene información sobre más de trescientas enfermedades. En su decimoséptima edición se han revisado de forma exhaustiva todas las enfermedades comprendidas en la edición anterior y se ha actualizado casi un tercio; se presenta también material adicional sobre las enfermedades víricas por Hendra y Nipah.

Gracias al nuevo formato electrónico, los usuarios podrán comprar la sección específica sobre la enfermedad que les interesa o suscribirse por un año al libro completo en formato electrónico.

Siga los tres pasos siguientes y de inmediato tendrá a su disposición El control de las enfermedades transmisibles en formato electrónico:

* Visite el sitio: http://www.ingentaselect.com

* Seleccione "Pan American Health Organization" en la opción Browse by publishers

* Seleccione el control de las enfermedades transmisibles, 17. ${ }^{\mathrm{a}}$ ed. 